\title{
Effect of Stress-Induced Anisotropy on the Noise of Ring-Core Fluxgate
}

\author{
Mattia Butta ${ }^{1}$, Pavel Ripka ${ }^{1}$, and Luděk Kraus ${ }^{2}$ \\ ${ }^{1}$ Department of Measurement, Faculty of Electrical Engineering, Czech Technical University in Prague, \\ Prague 166 36, Czech Republic \\ ${ }^{2}$ Institute of Physics, Academy of Sciences of the Czech Republic, Prague 110 00, Czech Republic
}

In this paper, we show how an anisotropy perpendicular to the direction of excitation is favorable to obtain low noise in fluxgate sensors. We produced ring cores by electroplating NiFe thin film $(6 \mu \mathrm{m})$ over a bended copper substrate. After the electroplating process, the sample was released and back-stress-induced anisotropy in the magnetic film due to magnetostriction of the material. As a result, we obtained rings with regions showing hard behavior and orthogonal regions showing soft behavior. Then, we used such rings as core for fluxgate and measured both noise and sensitivity for different orientation of the ring in the pick-up coil. When the pick-up coil covers the area with anisotropy perpendicular to the direction of excitation, the sensitivity is lower due to lower permeability, but finally, the noise is lower. On the other hand, rotating the core by $90^{\circ}$, the anisotropy becomes parallel to the direction of excitation and thus the sensitivity increases. Nevertheless, the noise also increases, showing that such anisotropy parallel to direction of excitation is unfavorable even if it brings higher sensitivity.

Index Terms-Anisotropy, fluxgate, nanocrystalline, noise, permalloy.

\section{INTRODUCTION}

$\mathbf{N}$ OISE of $f$ uxgate sensors is typically composed of several contributions: 1) the magnetic noise of the material used as a core of the sensor; 2) the noise of the demodulator used to extract the second harmonic from the voltage induced in the pick-up coil; and 3) the noise due to the excitation current and many others [1]-[4]. A precise design of the magnetometer can reduce most of these contributions and eventually the main source of noise is given by the intrinsic magnetic noise of the core.

Such noise is generated by Barkhausen noise, which occurs in the magnetic core when the magnetization is reversed from one saturation state to the opposite one, as periodically happens in fuxgate sensors. In order to reduce this, noise anisotropy with easy axis perpendicular to the direction of magnetization is desirable. It has been shown in fuxgate based on toroidal and race-track cores that such a change of anisotropy obtained with $f$ eld annealing on amorphous ribbons helps in reducing the noise [5]. In that case, the easy direction is perpendicular both to the direction of the excitation feld and sensing direction.

In this paper, we show the dependence of the noise in a f uxgate on the anisotropy direction when using ring cores with electroplated $\mathrm{NiFe}$ nanocrystalline $\mathrm{f} \mathrm{lm}$. In order to make such dependence evident, we manufactured a ring with an in-plane anisotropy in a linear direction by electroplating the ring under bending. Then, we employed such rings as core of f uxgates and measured both noise and sensitivity for different relative angle between the core and pick-up coil. It turned out that the noise is strongly correlated to the direction of magnetization.

Manuscript received June 12, 2014; revised August 9, 2014; accepted August 27, 2014. Date of current version January 26, 2015. Corresponding author: M. Butta (e-mail: mattia@butta.org).

Color versions of one or more of the fgures in this paper are available online at http://ieeexplore.ieee.org.

Digital Object Identifie 10.1109/TMAG.2014.2358793

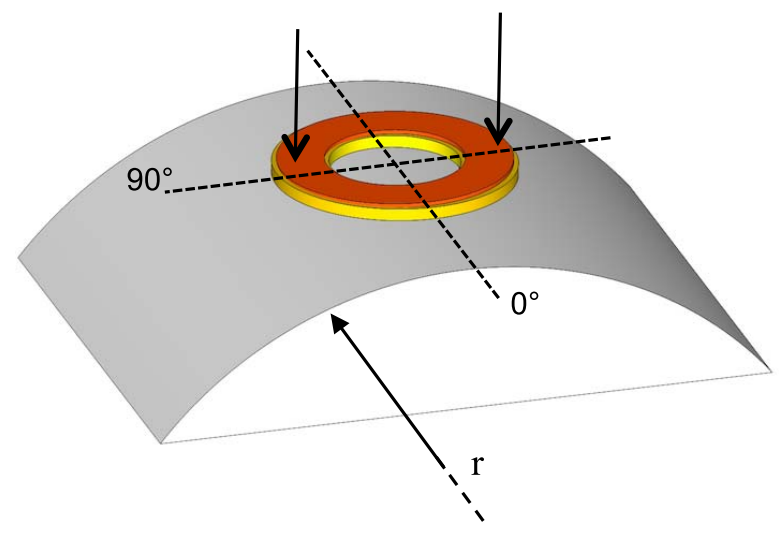

Fig. 1. Setup for bending of the ring during electroplating (not to scale). The ring is attached to the curved support by pressing it in the point shown by the arrows.

\section{SAMPLES PREPARATION}

The rings were manufactured by electroplating $\mathrm{NiFe} f \mathrm{~lm}$ over a copper layer. We used f berglass substrate with overlapping copper substrate. The copper $\mathrm{flm}$ was reduced to $9 \mu \mathrm{m}$ from the initial $18 \mu \mathrm{m}$ both to improve the roughness and reduce the thickness (signif cant conductive material inside the excitation coil can cause non-negligible eddy currents). The fil has been electroplated using a standard bath with the following composition: 1) $\left.\mathrm{FeSO}_{4} \cdot 7 \mathrm{H}_{2} \mathrm{O}(8 \mathrm{~g} / \mathrm{L}) ; 2\right) \mathrm{NiSO}_{4} \cdot 6 \mathrm{H}_{2} \mathrm{O}$ (125 g/L); 3) $\left.\mathrm{NiCl}_{2} \cdot 6 \mathrm{H}_{2} \mathrm{O}(20 \mathrm{~g} / \mathrm{L}) ; 4\right) \mathrm{H}_{3} \mathrm{BO}_{3}(40 \mathrm{~g} / \mathrm{L})$; and 5) saccharin $(6 \mathrm{~g} / \mathrm{L})$ in de-ionized water at $55{ }^{\circ} \mathrm{C}[6]$. The anode was composed of a platinum grid. The ring had $28 \mathrm{~mm}$ external diameter and $18 \mathrm{~mm}$ inner diameter.

In order to generate anisotropy in the electroplated film we bended the ring over a plastic support during the electroplating process (Fig. 1). At the end of the electroplating process, the ring was detached from the bending support and it retrieved its natural straight state. The radius of the bending support was $r=55 \mathrm{~cm}$. 


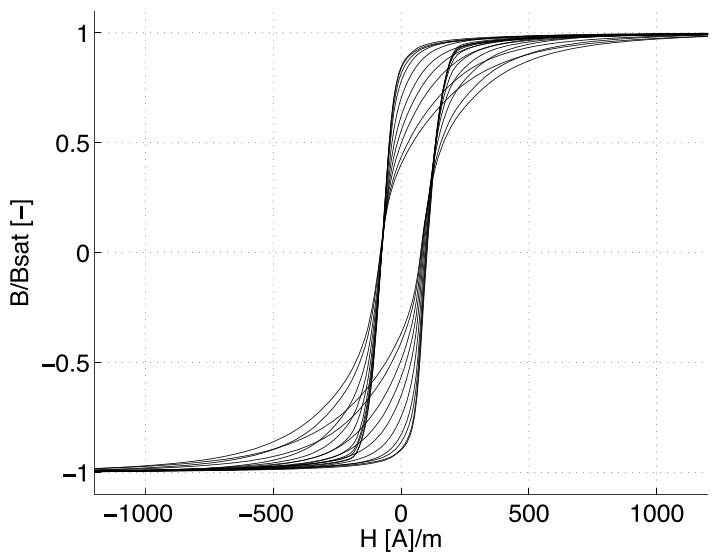

Fig. 2. Hysteresis curves of the electroplated ring measured by induction method moving the pick-up coil from $0^{\circ}$ to $90^{\circ}$ along the ring circumference (lowest permeability is $0^{\circ}$ and highest permeability is $90^{\circ}$ ).

In this way, a mechanical back-stress is permanently applied to the film which was electroplated in bended state. The current density used for the electroplating was $j=12 \mathrm{~mA} / \mathrm{cm}^{2}$ : this resulted in a magnetostrictive NiFe. The result of the back-stress on a magnetostrictive $\mathrm{flm}$ is the anisotropy with the easy axis in the direction of the stress [7], [8]. The bending angle is $2.9^{\circ}$, the resulting back-stress was $6.8 \mathrm{MPa}$.

We produced four samples with the same procedure under the same conditions, as well as we produced also a core with no bending during electroplating to allow us an easy comparison.

\section{Characterization OF The SAMPles}

In order to highlight the anisotropy, we measured the $B-H$ loops of all samples using conventional induction method. The excitation coil was wound around the whole circumference of the ring using $0.18 \mathrm{~mm}$ diameter copper wire (270 turns). The pick-up coil was composed of 30 turns of thin 0.1 diameter copper wire concentrated in $2 \mathrm{~mm}$ width. The pick-up coil could be moved along the circumference of the ring allowing us to measure the magnetic properties of different parts of the ring. The loops have been measured from $0^{\circ}$ to $350^{\circ}$ in steps of $10^{\circ}$. In Fig. 2, we show, for sake of clarity, only the loops from $0^{\circ}$ to $90^{\circ}$. We can clearly see that the fil has anisotropic behavior.

We can better see that the anisotropy is linear in Fig. 3, where we plotted the relative permeability of the rings obtained from the measurement of $B-H$ loop versus the angle along the whole circumference of the rings. The periodicity indicates that the ring has easy axis of magnetization approximately in $90^{\circ}-270^{\circ}$ axis, whereas the hard axis of magnetization is in $0^{\circ}-180^{\circ}$ axis

We should notice that section of the ring at $180^{\circ}$ angle shift is not identical. For instance, the peaks at maximum permeability at $90^{\circ}$ and $270^{\circ}$ have a non-negligible variance. We believe this is due to non-uniform bending of the ring due to non-perfect contact of the ring to the curved support.
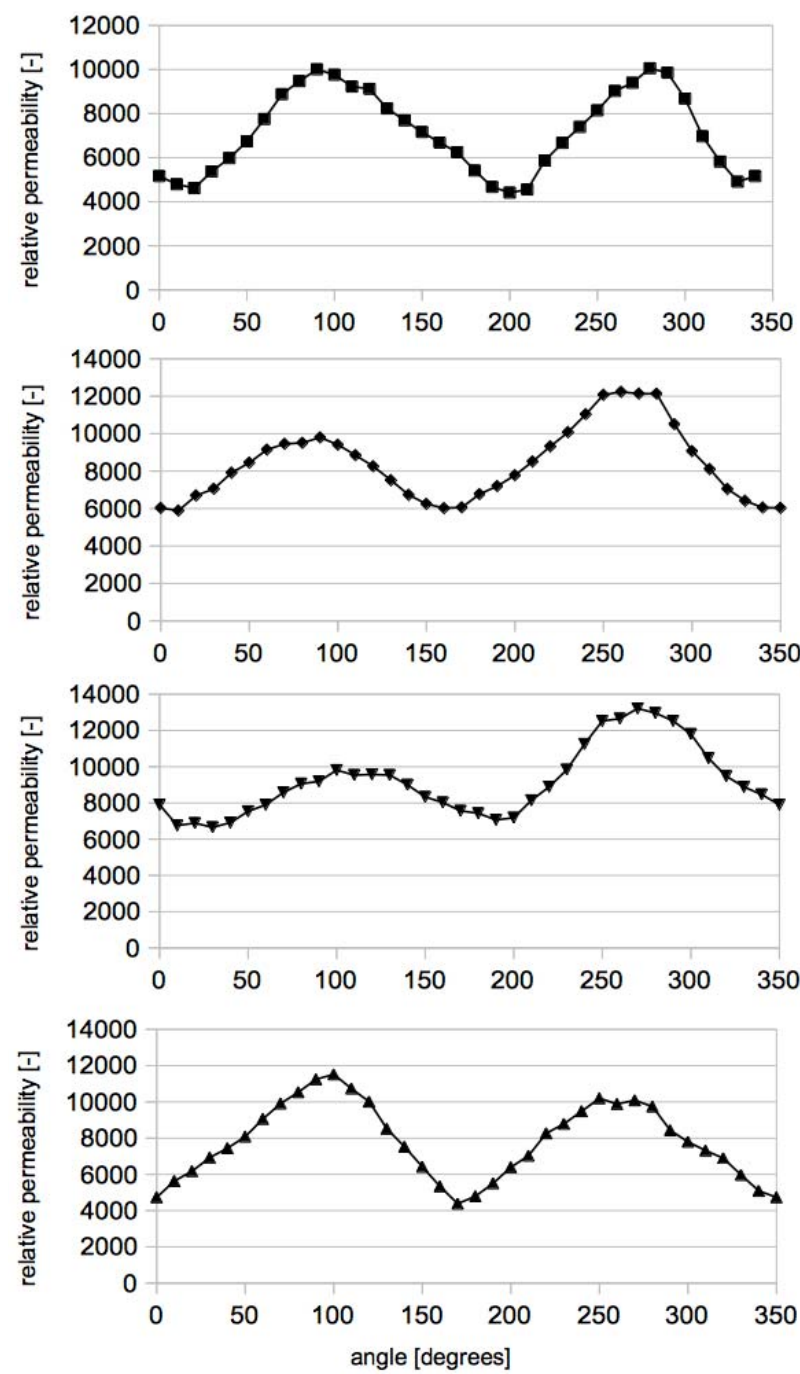

Fig. 3. Relative permeability of the electroplated $\mathrm{f} 1 \mathrm{~m}$ along the whole circumference of the ring for the four rings produced.

\section{Noise Measurement}

We then used the ring cores as core for f uxgates. The core was placed inside an 80 turn pick-up coil. The pick-up coil was $5 \mathrm{~mm}$ wide, less than typical fuxgate (as a rule of the thumb, the pick-up coil is equal to the internal diameter of the ring). We chose so narrow pick-up coil because we wanted to pick-up the fux from a narrow region of the ring. A larger coil would return us the f ux from areas of ring with different anisotropy averaging the effect of anisotropy on the noise and thus making more diff cult to recognize it

A parallel capacitor was used to tune the induced voltage of the pick-up coil to the second harmonic and thus to maximize the sensitivity. The second harmonic was demodulated using a Signal Recovery 7265 DSP lock-in amplif er. The excitation current was $1.9 A_{p-p}$, and frequency was $20 \mathrm{kHz}$. The current was sinusoidal and produced by a waveform generator and current buffer. Any undesired dc component of the current was removed using a series blocking capacitor.

We measured the sensitivity of such sensors in a Helmholtz coil rotating the core every $10^{\circ}$ : the resulting sensitivity for the four cores is plotted in Fig. 4. 


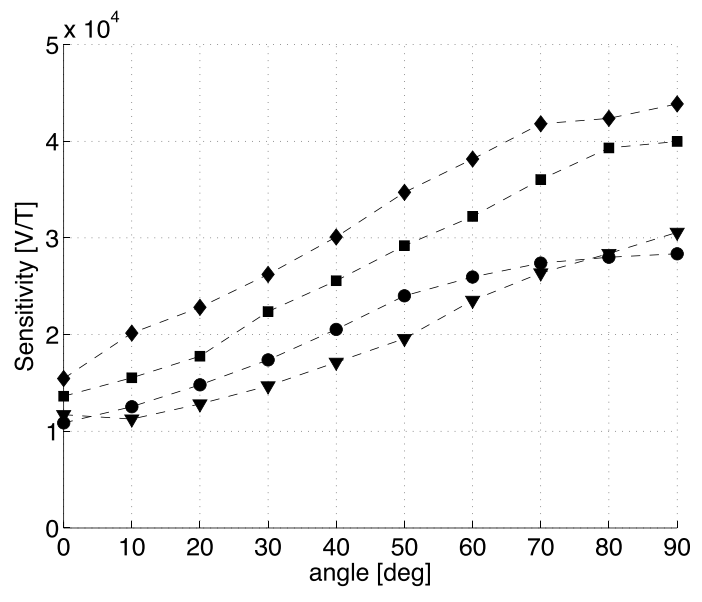

Fig. 4. Sensitivity of the second harmonic of four fluxgate based on the ring cores with stress-induced anisotropy, excited with $1.9 A_{p-p}$ current at $20 \mathrm{kHz}$ and tuned pick-up coil.

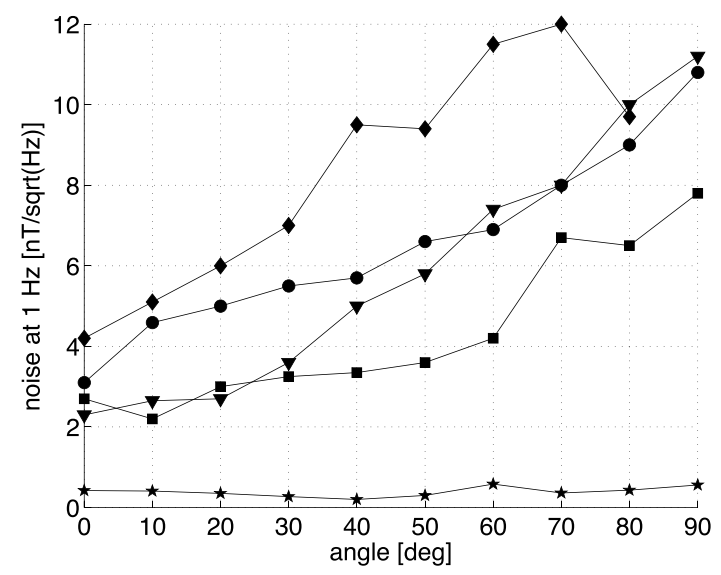

Fig. 5. Diamonds, circles, triangles, and squares: dependence of the noise spectral density at $1 \mathrm{~Hz}$ of the four sensors based on cores with stress-induced anisotropy versus angle relative to anisotropy direction. Stars: noise at $1 \mathrm{~Hz}$ of a sensor based on uniform core.

The sensitivity significa tly changes from sensor to sensor, however in all cases, the sensitivity strongly increases when moving from the region at $0^{\circ}$ corresponding to the hard axis of magnetization to the region at $90^{\circ}$ with easy axis of magnetization. While at $0^{\circ}$, the sensitivity is $\sim 10-15 \mathrm{kV} / \mathrm{T}$; at $90^{\circ}$, it becomes about three times larger. This is in accordance with the measurement of permeability, because the sensitivity of a fuxgate is directly connected to the permeability of its core [9].

Next, we measured the noise of the sensors to understand the effect of anisotropy on the noise. The sensor was placed in a four layer shielding and the noise spectrum acquired 10 times and averaged (rms averaging). The measurement of noise was repeated moving the core from $0^{\circ}$ to $90^{\circ}$ in steps of $10^{\circ}$ to test the dependence of the noise on the angle. The results are plotted in Fig. 5 for all four sensors. As usually done for f uxgates and generally for magnetic sensors, we consider the spectral density of noise at $1 \mathrm{~Hz}$.

In addition, in this case, the noise differs from sensor to sensor; more interestingly, the results show the increase of

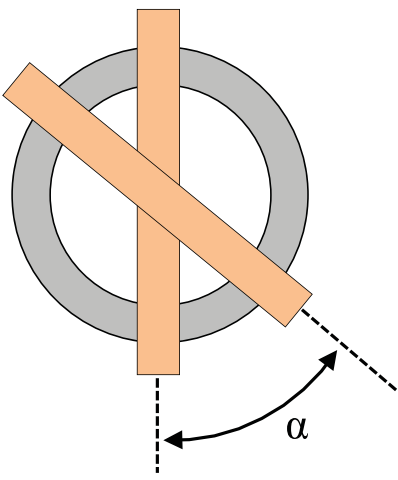

Fig. 6. Setup of the two coils used for measurement of correlation between the noise in different areas of the ring core.

noise as we rotate the core from $0^{\circ}$ to $90^{\circ}$ even if the sensitivity was increasing.

This conf rms that an anisotropy perpendicular to the direction of magnetization, such as the one we have on the $0^{\circ}-180^{\circ}$ axis, is favorable if we want low noise. Indeed, the lower noise is obtained when picking up the fux from the portion of the ring with harder axis, despite the sensitivity was there the lowest.

The dependence of the noise on the anisotropy direction is even clearer if we consider the noise of an extra ring electroplated without bending and therefore with uniform behavior all over its circumference. Its noise has been plotted in Fig. 5 along with the noise of the four rings electroplated under bending. In this case, the noise does not depend on the angle. There are some areas with larger noise (such as $60^{\circ}$ ) but this is most probably due to local defect of the magnetic $\mathrm{f} \mathrm{lm}$ in that region. No clear dependence of the noise on the angle can be observed in case of ring with uniform properties.

However, we must also note that this extra ring electroplated without bending not only has a noise, which is uncorrelated to the angle, but it has also much lower noise than the best region of the rings with induced anisotropy. If we consider the best condition for those four rings (that is, $0^{\circ}$ ), the noise is never $<2 \mathrm{nT} / \sqrt{ } \mathrm{Hz}$, and it increases up to $\sim 12 \mathrm{nT} / \sqrt{ } \mathrm{Hz}$ as we rotate the ring to easy axis of magnetization; this noise is in fact very large. On the contrary, the ring electroplated without bending shows a noise, which is one order of magnitude lower.

\section{Correlation of the Noise}

In order to understand this behavior, we measured the correlation between the noise originated in different regions of the ring. We wound two pick-up coils to the sensor shifted by an angle $\alpha=30^{\circ}, 60^{\circ}$, or $90^{\circ}$ (Fig. 6).

The two output voltages of the two pick-up coils have been both tuned with a capacitor and they have been independently demodulated by two lock-in amplif ers, the Signal Recovery 7265 used before and an additional Stanford Research 830 lock-in amplifie. Then, we measured the correlation between the two second harmonics extracted from the lock-in amplif er using an Agilent 35670A spectrum analyzer.

Using two completely different lock-in amplifie s to extract the second harmonics from the pick-up coils, we can be sure 


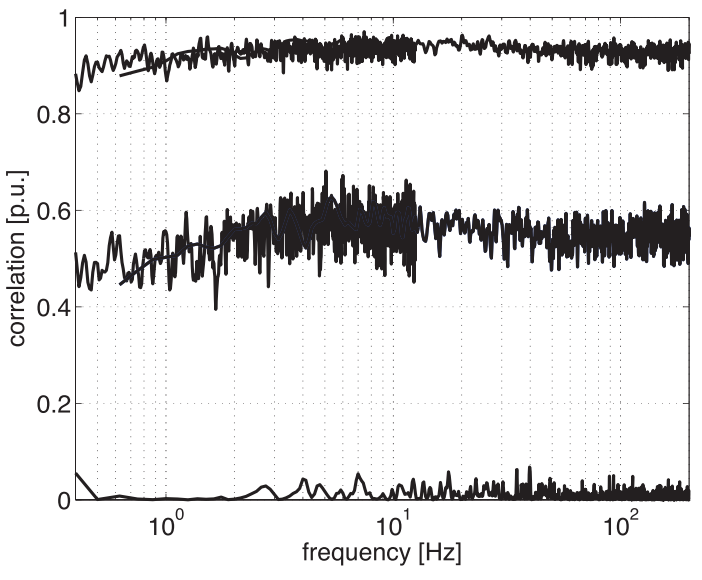

Fig. 7. Correlation between the second harmonics obtained from the pick-up coils depicted in Fig. 6 for $\alpha=30^{\circ}$ (highest), $60^{\circ}$ (medium), and $90^{\circ}$ (lowest).

that any possible correlation between the signals is not given by the conditioning electronics.

The correlation has been measured in a range between 0.4 and $200 \mathrm{~Hz}$. The results are shown in Fig. 7 for $30^{\circ}$, $60^{\circ}$, and $90^{\circ}$ angle between the pick-up coils.

We can see that there is a strong correlation $(\sim 0.9)$ if the coils are rotated by $30^{\circ}$. The correlation decreases, but it is still large $(0.55)$ if the angle is $60^{\circ}$, while falls to almost zero only at $90^{\circ}$.

This indicates that the noise originating from the region with easy axis of magnetization is not limited to that region, but it spreads to the neighboring areas of the ring.

In other words, the portion of the ring, which has high permeability, undergo a sudden reversal of the magnetization, which is inf uencing also other parts of the ring with harder behavior.

\section{CONCLUSION}

By electroplating NiFe thin $\mathrm{flm}$ under bending, we were able to induce anisotropy in the desired direction to the magnetic $\mathrm{flm}$. We have shown that anisotropy perpendicular to the direction of magnetization is favorable from the point of view of the noise. Nevertheless, the proposed method, while from one side, induces anisotropy in the proper direction in a region of the fil ; on the other side, it creates anisotropy in the wrong direction in the area of the ring rotated by $90^{\circ}$. Unfortunately, the excessive noise originating in this region spreads also to the adjacent areas of the ring biasing them and thus increasing the noise to unacceptable level even in the best regions. Therefore, it is not advisable to induce anisotropy orthogonal to direction of excitation by electroplating under bending because it unavoidably creates also an anisotropy parallel to direction of excitation in other areas of the ring and this is non-negligible.

We conclude that in order to effectively reduce the noise, it is vital to have a ring with anisotropy perpendicular to the direction of excitation all over the ring, that is, radial anisotropy.

\section{ACKNOWLEDGMENT}

This work was supported by the Grant Agency of the Czech Republic under Grant P102/12/2177.

\section{REFERENCES}

[1] O. V. Nielsen et al., "Analysis of a fuxgate magnetometer based on metallic glass sensors," Meas. Sci. Technol., vol. 2, no. 5, pp. 435-440, 1991.

[2] B. Narod, J. R. Bennest, J. O. Stromolse, F. Nezil, and R. A. Dunlap, "An evaluation of the noise performance of $\mathrm{Fe}, \mathrm{Co}, \mathrm{Si}$, and $\mathrm{B}$ amorphous alloys in ring-core fluxgat magnetometers," Can. J. Phys., vol. 63, no. 11, pp. 1468-1472, 1985.

[3] F. Primdahl, B. Hernando, O. V. Nielsen, and J. P. Petersen, "Demagnetising factor and noise in the fuxgate ring-core sensor," J. Phys. E, Sci. Instrum., vol. 22, no. 12, pp. 1004-1008, 1989.

[4] L. C. de Carvalho Benyosef, G. C. Stael, and M. Bochner, "Optimization of the magnetic properties of materials for f uxgate sensors," Mater. Res., vol. 11, no. 2, pp. 145-149, 2008.

[5] P. Butvin et al., "Field annealed closed-path f uxgate sensors made of metallic-glass ribbons," Sens. Actuators A, Phys., vol. 184, pp. 72-77, Sep. 2012.

[6] H. L. Seet, X. P. Li, Z. J. Zhao, Y. K. Kong, H. M. Zheng, and W. C. Ng, "Development of high permeability nanocrystalline permalloy by electrodeposition," J. Appl. Phys., vol. 97, no. 10, pp. 10N304-1-10N304-3, May 2005.

[7] Z. K. Wang, E. X. Feng, Q. F. Liu, J. B. Wang, and D. S. Xue, "Tuning stress-induced magnetic anisotropy and high frequency properties of FeCo fil s deposited on different curvature substrates," Phys. B, Phys. Condens. Matter, vol. 407, no. 18, pp. 3872-3875, 2012.

[8] M. Butta and L. Kraus, "Stress-induced anisotropy in electroplated FeNi racetrack f uxgate cores," IEEE Trans. Magn., vol. 50, no. 4, pp. 1-4, Apr. 2014.

[9] P. Ripka, Ed., Magnetic Sensors and Magnetometers. Norwood, MA, USA: Artech House, Dec. 2000. 